\title{
On the Fringe of Awareness: The Glance-Look Model of Attention-Emotion Interactions
}

\author{
$\mathrm{Li} \mathrm{Su}^{1}$, Philip Barnard ${ }^{1}$, and Howard Bowman ${ }^{2}$ \\ ${ }^{1}$ MRC Cognition and Brain Sciences Unit, Cambridge, United Kingdom \\ \{Li.Su, Phil.Barnard\}@mrc-cbu.cam.ac.uk \\ ${ }^{2}$ Centre for Cognitive Neuroscience and Cognitive Systems \\ and School of Computing, University of Kent, Canterbury, United Kingdom \\ H. Bowman@kent.ac.uk
}

\begin{abstract}
In previous work, we have developed a "Glance-Look" model, which has replicated a broad profile of data on the semantic Attentional Blink (AB) task and characterized how attention deployment is modulated by emotion. The model relies on a distinction between two levels of meaning: implicational and propositional, which are supported by two corresponding mental subsystems. The somatic contribution of emotional effects is modeled by an additional body-state subsystem. The interaction among these three subsystems enables attention to oscillate between them. Using this model, we have predicted the pattern of conscious perception during the $\mathrm{AB}$ and the changes of awareness when emotional or other task irrelevant processing occurs. We provide a specific account of the interaction between attention, emotion and consciousness. In particular, the dynamics of two modes of attending to meaning (implicational being more distributed and propositional being evaluative and specific) give rise to fringe awareness.
\end{abstract}

Keywords: fringe awareness, Attentional Blink, emotion, Glance-Look model, consciousness, body-state.

\section{Introduction}

The relationship between consciousness and attention is one of the most controversial issues in psychology, philosophy and cognitive neuroscience. One key debate is centered on whether or not attention and consciousness can be untangled into separate processes. A paradigm called the Attentional Blink (AB) [1] has demonstrated a situation where the same sensory input can lead to both conscious and non-conscious perception. The task involves targets being presented using Rapid Serial Visual Presentation (RSVP) at around ten items a second. The identification of a second target is impaired when it is closely preceded in time $(<500 \mathrm{~ms})$ by a first target. In addition, findings suggest that attention and consciousness are not only separable, but may also oppose each other. For instance, it has been discovered that task manipulations and emotional states can attenuate blink effects (i.e. enhance the awareness of the second target) by, it is argued, encouraging a more distributed state of attention, e.g. by using music, positive affect or dynamic visual patterns to 
counteract an overinvestment of attention [2,3,4]. Thus, in this context, reducing attentional focus seems to improve awareness. Hence, the $\mathrm{AB}$ paradigm provides a solid platform for investigation of the relationship between attention, emotion and conscious perception. This paper shows how our previously developed "GlanceLook" model $[5,6,7]$ can also provide a general information processing account for the interactions between attention, emotion, consciousness, and fringe awareness, as well as, more specifically, explaining overinvestment findings in the AB.

\section{The "Glance-Look" Model}

\subsection{Key-Distractor Attentional Blink Task}

The "Glance-Look" model, as shown in Figure 1, was first proposed to explain a variant of the $\mathrm{AB}$ paradigm, in which words were presented at fixation in RSVP format and targets were only distinguishable from background items in terms of their meaning. Participants were simply asked to report a word if it refers to a job or profession, such as "waitress", and these targets were embedded in a list of background words that all belonged to the same category, e.g. nature words. Participants could report what they believed was the target word's identity (Correct ID), say "Yes" if they were confident a job word had been there, but could not say exactly what it was (to capture a degree of awareness of meaning), or say "No" if they did not see a target, and there were, of course, trials on which no target was presented. However, streams also contained a key-distractor item, which, although not in the target category, was semantically related to that category, such as "tourist" or "husband" [8], or emotionally charged, such as "cancer" [9]. The serial-position that the target appeared after the key-distractor was varied. The effect of attentional capture is encapsulated in the serial position curve in Figure 2(A). We call this the key-distractor $\mathrm{AB}$ task.

\subsection{Basic Structure of the "Glance-Look" Model}

The key principles that underlie the "Glance-Look" model are as follows. 1) Items are composed of several constituent representations (CRs) passed through a pipeline from the visual system to the response system. On every cycle, a new CR enters the pipeline and all CRs currently in transit are pushed along one place. In this sense, it could be viewed as an analogue of a sequence of layers in a neural network, e.g. a synfire chain [10]. 2) The processing of meaning is divided into two stages, which are supported by the implicational (Implic) and propositional (Prop) subsystems. Each subsystem assesses a different type of meaning using Latent Semantic Analysis [11], a statistical learning technique, related to Principle Component Analysis and Hebbian learning. 3) A body-state subsystem monitors the outputs of Implic and feeds back emotional information in the form of "somatic markers" [12]. 4) Only one subsystem can be attended at a time, and it is only when attention is engaged at a subsystem that it can assess the salience of items passing through it. All unattended subsystems in the model process stimuli in parallel, in a kind of "zombie" mode, similar to that suggested by Crick and Koch [13]. We argue that it is attention and the interaction between these subsystems that brings information into consciousness. 


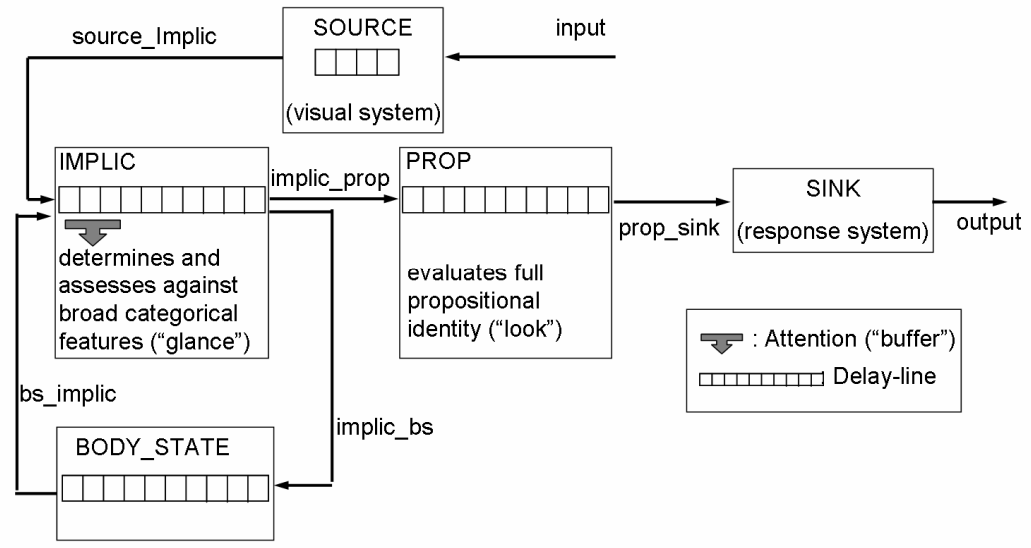

Fig. 1. The top-level schematic diagram of the "Glance-Look" model. Names in uppercase are processes, e.g. IMPLIC, names in lowercase are communication channels, e.g. implic_prop. IMPLIC, PROP, BODY_STATE, SOURCE, and SINK represent implicational subsystem, propositional subsystem, body-state subsystem, visual system, and response system respectively.

(A)

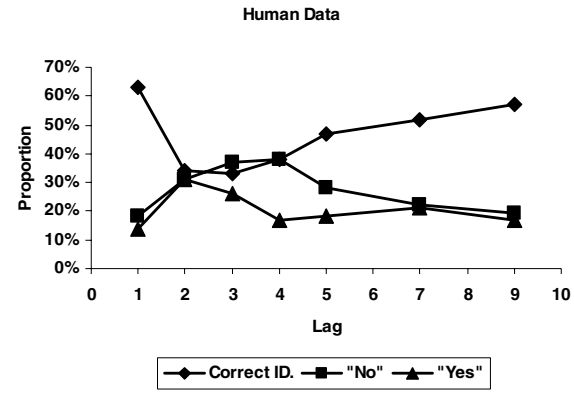

(B)

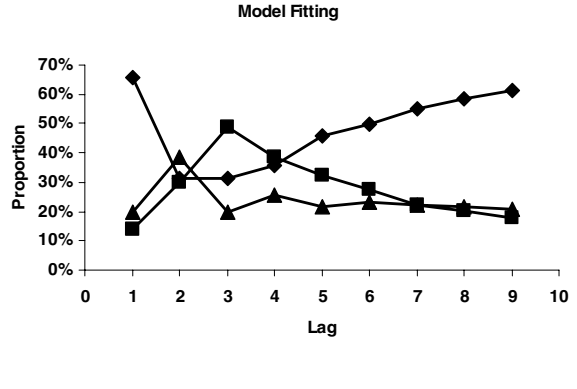

Fig. 2. (A) Proportion of different types of responses from humans [8]. Lag indicates the number of items intervening between the key-distractor and the target. (B) Model simulates the behavioral curves of the $\mathrm{AB}[5]$.

\subsection{How the Model Blinks}

In the context of the $\mathrm{AB}$, attention is captured when a key-distractor is interpreted as implicationally salient, as it is task or personally relevant. This then causes attention to be redeployed to Prop, in order to enable a more detailed assessment of the salience of the key-distractor. This redeployment of attention leaves a temporal window in which implicational salience is not fully assessed. In particular, the meaning of a target word can be processed to three different degrees, which, we argue, reflect 
different degrees of awareness and thus lead to three types of response, one of which provides a computationally explicit characterization of fringe awareness. Words that are fully (i.e. both implicationally and propositionally) interpreted can be (fully) consciously accessed and reported correctly with their identity. Some targets can be only partially processed, i.e. insufficient CRs have been assessed (by Prop), resulting in fringe awareness, reflecting the "Yes" response. This only happens during the transition between complete conscious and unconscious states. As shown in Figure 2, the results of the model simulation were comparable to human performance, with fringe awareness particularly evident at lag 2. Finally, some targets can be implicationally totally un-interpreted reflecting complete unawareness of the presence of target words, i.e. the "No" responses. This state is most likely to occur around the deepest points in the blink.

\subsection{The "Glance-Look" Account of the Attenuation Effect}

As previously discussed, there is evident that distracting participants can reduce blink depth. Consequently, overinvestment theories of the $\mathrm{AB}$ have become prominent. The degree of, distraction-induced, attenuation reported in $[2,3,4]$ should, though, reflect two factors: the degree to which the ancillary task has direct consequences for the representation of generic (implicational) meaning and the extent to which the reporting of an item requires extended evaluation of propositional meanings in our second stage. To elaborate further, in a typical laboratory setting, participants are encouraged to recall as accurately as possible. As previously argued by several authors $[2,3,4]$, this could well result in rather more investment of attention than is strictly necessary to accomplish item report. This hypothesis is consistent with our model, in the sense that, overinvestment may reflect extended processing in our second "propositional" stage, where attention may take a long time to switch back to a state in which implicational representations are attended. The implicational mode of attending to meaning has a broader focus on generic meaning, which may incorporate affect, and derivatives of multimodal or lower order inputs, such as music. When subjects are exposed to dynamic patterns, being visual, musical or internally generated, while performing the central AB task, there would be more changes in input to implicational meaning. With our model of distributed control, these may well encourage the implicational mode of attending to meaning, and support more distributed awareness of this type of generic meaning. Crucially, the paradigm often involves reporting letters in a background stream of digits $[2,3,4]$. Letters are drawn from a small and highly familiar set, and hence, in the limit, this may require only the briefest "look" at a propositional representation to support correct report.

Attenuation should be less pronounced either with secondary tasks whose content does not directly influence the level of generic (implicational) meaning or, as with semantic blink effects, where a fuller evaluation of propositional meanings is required. Should such effects be found, it would provide an encouraging convergence between basic laboratory tasks and the literature on attention to meaning and affect in emotional disorders, using a non-computationally specified version of our current proposal [14]. 


\section{Modeling Conscious Perception during the AB}

The "Glance-Look" model takes specific account of the interaction between attention, emotion and consciousness. Firstly, we have shown that lack of awareness can be accounted for by the allocation of attention to different levels of meaning in a system where there is only distributed control of processing activity. Just as the focus of our attention may shift among entities in our visual and auditory scenery under the guidance of salient change, shifts in attention to different entities in our semantic scenery can lead to RSVP targets being either, 1) correctly identified; 2) giving rise to a fringe awareness of presence; or 3) overlooked. Salience states at each of two levels of meaning allow these three response patterns to be captured. Although the proposal, like that of Chun and Potter [15], relies on two stages, both of our stages are semantic in nature and the temporal dynamic involves controlled changes in the focus of attention, rather than classic capacity or resource limitations. The idea of monitoring a generic form of meaning for implicational salience, the level at which affect is represented in the model, and switching only when required to evaluate propositional meaning, represent two "modes" of attending to meaning. The former mode has a broader focus on generic meaning (i.e. the "gist") and the latter a more evaluative focus on specific meanings, which can be verbally reported. This is similar to the distinction in the literature between "phenomenal" and "access" awareness [16]. Furthermore, the broader mode of processing meaning bears some resemblance to recent suggestions that task manipulations can attenuate blink effects, by encouraging a more distributed state of awareness, which would arise at our implicational level. In particular, music, positive affect or dynamic visual patterns may counteract the overinvestment of attention [2,3,4] and produce a fleeting conscious percept [13].

\section{Conclusions}

In summary, consciousness is modeled as an emergent property from the interaction among three subsystems: implicational, propositional and body-state. In particular, we differentiate two types of consciousness. One is akin to full "access" awareness, i.e. conscious content can be verbally reported, and is supported by both implicational and propositional processing. In other words, it is a result of a detailed "look" and more extensive mental processing. The other is akin to "phenomenal" (or fringe) awareness, and lacks the capacity of linguistic access. We argue that the latter is a result of attending to the implicational level or "glance". It is also notable that the implicational level is holistic, abstract and schematic, and is where multimodal inputs are integrated, and affect is represented and experienced [17].

In addition, the "Glance-Look" model makes several predictions on the relationship between these two modes of consciousness. First, fringe awareness provides a basis for a more complete state of consciousness. Second, comparing to full access awareness, phenomenal or fringe awareness is directly affected by emotional, multimodal, bodystate and lower order inputs. However, once propositional level information has been attended, a conscious percept is much less likely to be interrupted. The validation of these predictions awaits further experimental work. 


\section{References}

1. Raymond, J.E., Shapiro, K.L., Arnell, K.M.: Temporary suppression of visual processing in an RSVP task: an attentional blink. Journal of Experimental Psychology: HPP 18(3), 849-860 (1992)

2. Arend, I., Johnston, S., Shapiro, K.: Task irrelevant visual motion and flicker attenuate the attentional blink. Psychonomic Bulletin and Review 13(3), 600-607 (2006)

3. Olivers, C.N.L., Nieuwenhuis, S.: The beneficial effects of concurrent task: irrelevant mental activity on temporal attention. Psychological Science 16, 265-269 (2005)

4. Olivers, C.N.L., Nieuwenhuis, S.: The beneficial effects of additional task load, positive affect, and instruction on the attentional blink. Journal of Experimental Psychology 32, 364-379 (2006)

5. Su, L., Bowman, H., Barnard, P.J.: Attentional Capture by Meaning, a Multi-level Modelling Study. In: 29th Annual Meeting of the Congitive Science Society, pp. 15211526 (2007)

6. Su, L., Bowman, H., Barnard, P.J., Wyble, B.: Process algebraic modelling of attentional capture and human electrophysiology in interactive systems. Formal Aspects of Computing 21(6), 513-539 (2009)

7. Bowman, H., Su, L., Wyble, B., Barnard, P.J.: Salience Sensitive Control, Temporal Attention and Stimulus-Rich Reactive Interfaces. In: Human Attention in Digital Environments. Cambridge University Press, Cambridge (2010)

8. Barnard, P.J., Scott, S., Taylor, J., May, J., Knightley, W.: Paying attention to meaning. Psychological Science 15(3), 179-186 (2004)

9. Barnard, P.J., Ramponi, C., Battye, G., Mackintosh, B.: Anxiety and the deployment of visual attention over time. Visual Cognition 12(1), 181-211 (2005)

10. Abeles, M., Bergman, H., Margalis, E., Vaadia, E.: Spatiotemporal firing patterns in the frontal cortex of behaving monkeys. Journal of Neurophysiology 70, 1629-1638 (1993)

11. Landauer, T.K., Dumais, S.T.: A Solution to Plato's Problem: The Latent Semantic Analysis Theory of the Acquisition, Induction and Representation of Knowledge. Psychological Review 104, 211-240 (1997)

12. Damasio, A.: Descartes' Error. G.P. Putnam's Sons, New York (1994)

13. Crick, F., Koch, C.: A framework for consciousness. Nat. Neur. 6, 119-126 (2003)

14. Teasdale, J.D.: Emotional processing, three modes of mind and the prevention of relapse in depression. Behaviour Research and Therapy 37, S53-S77 (1999)

15. Chun, M.M., Potter, M.C.: A two-stage model for multiple target detection in rapid serial visual presentation. Journal of Experimental Psychology: HPP 21(1), 109-127 (1995)

16. Lamme, V.A.: Why Visual Attention and Awareness are Different. Trends in Cognitive Science 7, 12-18 (2003)

17. Barnard, P.J.: Interacting cognitive subsystems: modelling working memory phenomena with a multi-processor architecture. In: Miyake, A., Shah, P. (eds.) Models of Working Memory, pp. 298-339. Cambridge University Press, Cambridge (1999) 